\title{
A 61-year-old woman with chronic leg lymphedema managed with complete decongestive therapy
}

\author{
Larysa Bondalevich MD, Stefan de Laplante MD
}

- Cite as: CMAJ 2020 June 29;192:E708-12. doi: 10.1503/cmaj.190227

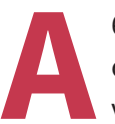

61-year-old woman presented to her family physician's office with long-standing bilateral leg swelling. She voiced that her legs had become "heavy" and that the skin was "thick" and "burning." She had started using a cane for gait instability.

An insidious onset of swelling in her left lower leg had started more than 25 years earlier without any obvious cause. A diuretic was initially prescribed; however, the swelling progressed. The patient was subsequently prescribed various diuretic dosing regimens, with minimal improvement. The diuretic was eventually stopped 3 years before the current presentation.

Over the years, the patient's right lower leg had also begun to swell. She had undergone venous Doppler ultrasonography of the peripheral extremities 16 years earlier to rule out venous pathologies and was evaluated for liver disease and congestive heart failure. The results of the investigations were negative.

The patient's medical history included overactive bladder and osteoarthritis. She had no history of coronary artery disease, venous thrombosis or liver disease. There was no family history of lymphedema. She was taking solifenacin $5 \mathrm{mg} / \mathrm{d}$ for overactive bladder and a calcium supplement.

\section{KEY POINTS}

- Chronic lymphedema is an underdiagnosed and undertreated condition; clinicians should consider the diagnosis when a patient has persistent extremity swelling.

- Morbidity associated with chronic lymphedema can be managed successfully with complete decongestive therapy when the treatment is provided by a trained therapist and the patient is adherent to the treatment and prescribed self-management routine.

- Untreated lymphedema can cause severe physical impairment and psychological distress and can have serious complications.

On physical examination, the patient had bilateral nonpitting leg edema (Figure 1). The left lower leg was larger than the right, and the ankle anatomy was obscured on visual inspection. The skin of both lower legs was thick and rigid on palpation, with deep skin creases and areas of hyperkeratosis, and there was mycosis of the left first toenail. Stemmer sign (Box 1) was positive. There were no signs of venous thrombosis. Bilateral leg ultrasonography showed normal and competent peripheral veins.

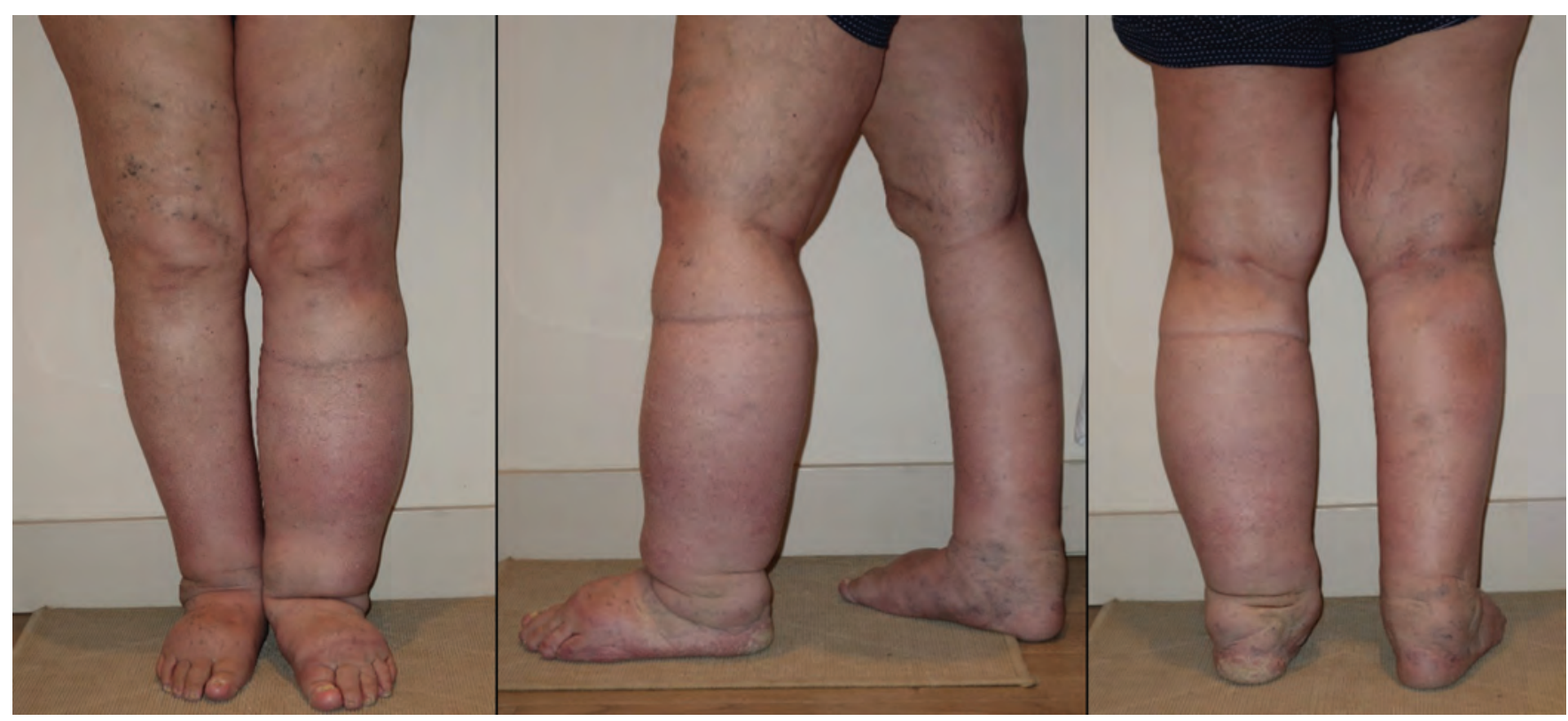

Figure 1: Anterior, lateral and posterior view of both legs in a 61-year-old woman with bilateral chronic lymphedema, before treatment with complete decongestive therapy. 


\section{Box 1: Lymphedema classification ${ }^{1-3}$}

\section{Classification}

- Primary: lymph transport impaired from dysplasia of the lymphatic system (idiopathic)

- Secondary: edema with a known cause (e.g., after a lymph node excision; after radiotherapy; obesity; filariasis; malignant, posttraumatic or inflammatory causes)

\section{Stages}

The stages of lymphedema are usually differentiated according to the extent of swelling:

- Stage 0 , latent: lymph stasis, no clinical signs of lymphedema

- Stage 1: spontaneously reversible lymphedema, subsides with limb elevation; pitting is present

- Stage 2: spontaneously irreversible edema, less pitting; moderate or pronounced fibrosis, positive Stemmer sign;* beginning of abnormal fat deposits

- Stage 3: chronic skin alterations (hyperkeratosis, pachydermia, fat deposits, papillomatosis, hyperpigmentation); marked subcutaneous fibrosis or sclerosis; pitting is absent; known as lymphostatic elephantiasis

*Positive Stemmer sign: inability to pick up a fold of thickened skin at the base of the second toe (fibrosis with or without abnormal fat is already present; lymphedema is at least stage 2). Normal thickness of skin fold at the second toe is $2-4 \mathrm{~mm}$.

\section{Box 2: Complete (or combined or complex)} decongestive therapy ${ }^{1,3}$

\section{Components}

- Manual lymph drainage

- Compression

- Skin care

- Physical exercise

\section{Phases of treatment}

Phase 1: intensive (decongestive) phase

- Daily treatments with manual lymph drainage and compression bandaging by a trained lymphedema therapist until the affected body part is decongested

- Duration varies with the severity of the condition, from 2 to 8 weeks

- Goal: edema reduction with manual lymph drainage and compression bandaging

Phase 2: maintenance phase

- Compression garments, exercise and skin care

- Manual lymph drainage, when required

- Goal: conservation and optimization of the results achieved during the intensive phase

The patient was assessed by a vascular surgeon, who obtained a lymphangiogram; it confirmed the presence of lymphedema. Initial management with compression bandaging performed by a community care agency, followed by graded elastic knee-high 20-30 mm Hg compression stockings, provided very minimal benefit.

The patient was referred to a certified lymphedema therapist. A course of complete decongestive therapy (Box 2) was initiated. Phase 1 began with daily 60-minute manual lymph drainage sessions

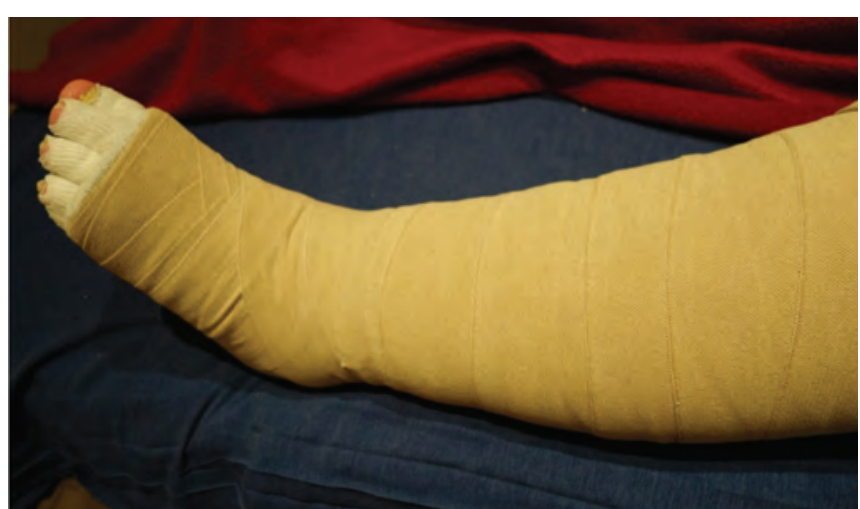

Figure 2: Multilayered short-stretch compression bandaging applied to the left leg.

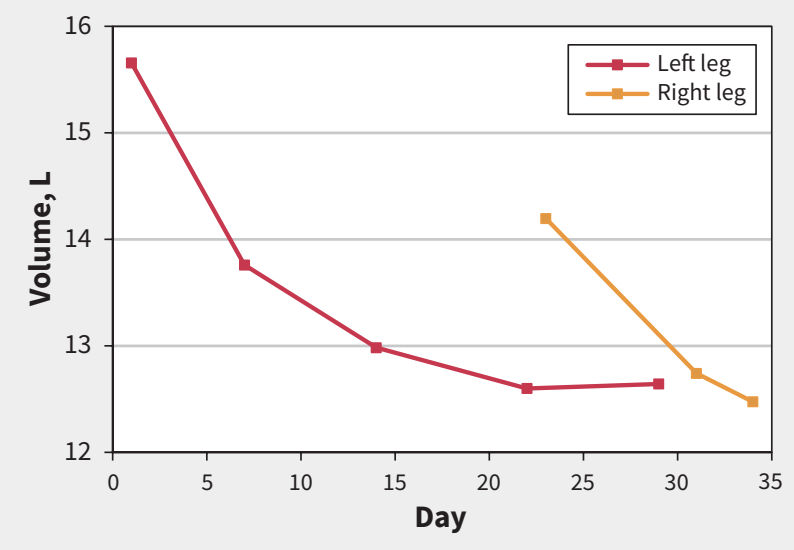

Figure 3: Progress of the leg-volume reduction in both legs during the course of treatment in a 61-year-old woman with bilateral chronic lymphedema.

for the left leg, followed by compression bandaging (Figure 2). The treatments were performed 5-6 times per week. The patient also performed prescribed decongestive exercises every day. Standardized circumference measurements taken in 4-cm increments were used to calculate each limb's volume (Kuhnke method ${ }^{1}$ ) and monitor its reduction (Figure 3 ).

The intensive phase lasted 3 weeks for the left leg and 2 weeks for the right. A substantial volume reduction of $4.735 \mathrm{~L}$ (left leg $3.014 \mathrm{~L}$; right leg $1.721 \mathrm{~L}$ ) was observed during the treatment, corresponding to a 4.5-kg weight loss (Figure 4; Appendix 1, available at www.cmaj.ca/lookup/suppl/doi:10.1503/ cmaj.190227/-/DC1). As a result of the treatment, the skin condition of the legs improved, and the fibrotic tissue softened. The patient also reported alleviation of "burning" and heaviness in the legs, as well as notable improvement in her mobility.

On reaching the maximum volume reduction, the patient was prescribed custom-made flat-knitted (inelastic) compression stockings (40 $\mathrm{mm} \mathrm{Hg}$ for the left leg and $30 \mathrm{~mm} \mathrm{Hg}$ for the right). She was also taught self-bandaging and exercises for the maintenance phase.

The follow-up appointment 24 months later showed that the bilateral leg volume was well controlled by the patient wearing compression garments $14-16$ hours a day and performing the 


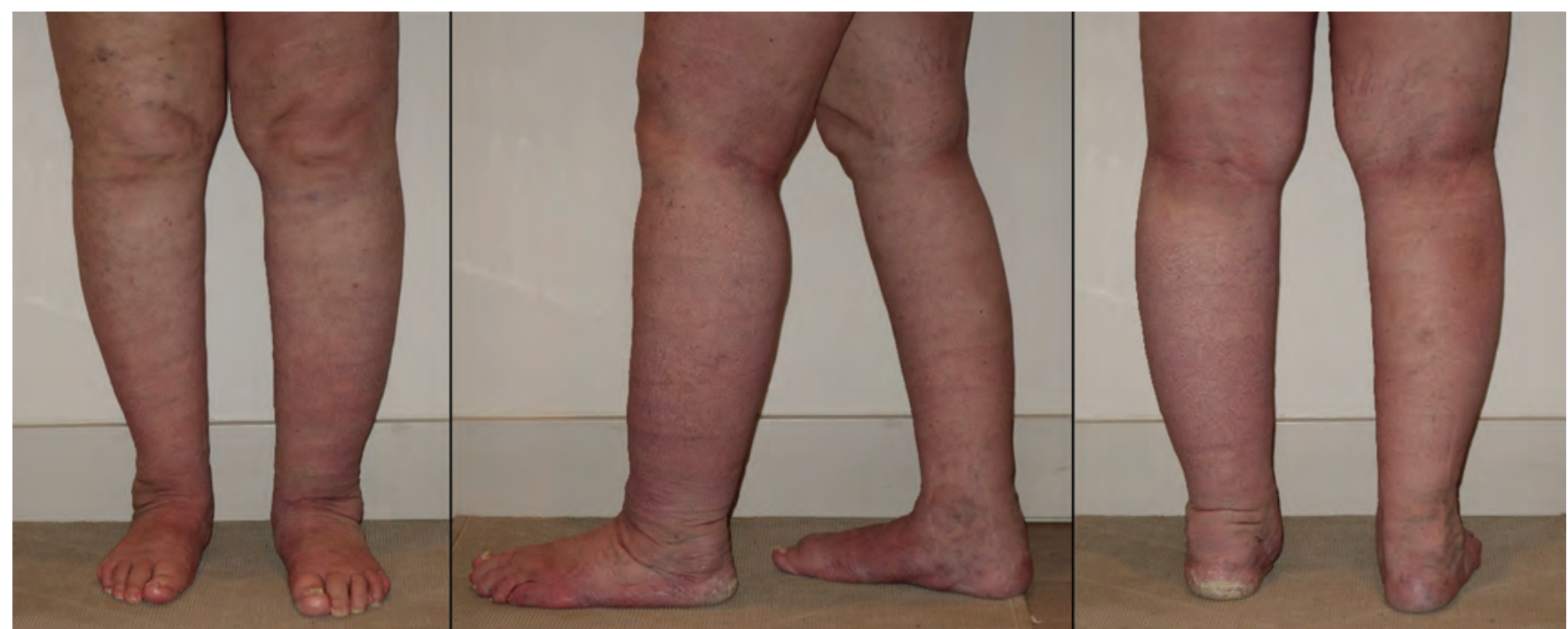

Figure 4: Anterior, lateral and posterior view of the legs upon completion of 2 months of complete decongestive therapy.

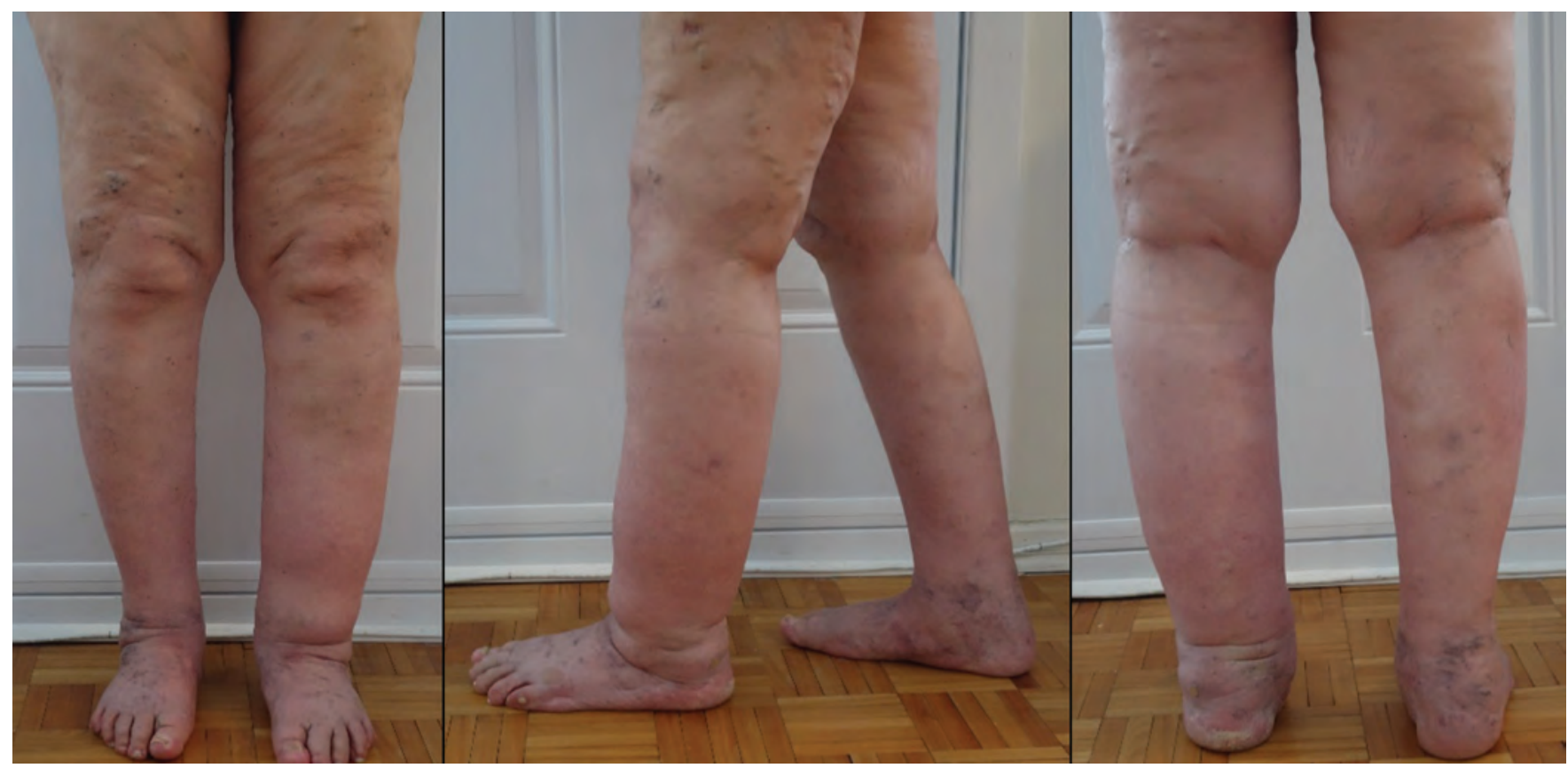

Figure 5: Anterior, lateral and posterior view of the legs 2 years after the intensive phase of complete decongestive therapy.

prescribed exercises (Figure 5; Appendix 1). The patient reported a lingering sensation of discomfort and heaviness in her legs. On examination, the skin of the lower legs appeared thick and rigid on palpation, although the patient thought there was some improvement over baseline.

\section{Discussion}

Primary lymphedema is an uncommon disease caused by impaired lymph drainage from developmental, presumably genetic, defects of the lymph vessels or lymph nodes. Secondary lymphedema is usually a consequence of damage to lymph-conducting pathways (Box 2). ${ }^{4,5}$

Prevalence estimates for primary lymphedema reported by different sources vary from $1-1.15$ in 100000 individuals ${ }^{6,7}$ to 1 in
6000 at birth. ${ }^{8}$ Primary lymphedema most commonly involves the lower limbs (as in our patient), but it can also affect the arms, trunk, genitals, head and neck. ${ }^{4,6}$ It is reported more often in women than in men. ${ }^{4,6}$

The number of individuals affected by all forms of lymphedema is growing, with an estimated 300000 people with this chronic condition in Canada and 200 million worldwide. ${ }^{6,8}$

\section{Pathophysiology and clinical manifestations}

Lymphatic vessels maintain tissue fluid balance by returning the capillary ultrafiltrate and filtered plasma proteins from the interstitium to the bloodstream, working as a coordinated unit with blood capillaries. When the lymphatic system fails to provide adequate drainage, accumulation of protein-rich fluid in the interstitial 
Box 3: Complications of lymphedema ${ }^{1,9}$

- Cellulitis* (erysipelas), septicemia

- Fibrosis*

- Fungal infections (e.g., nail mycosis, tinea pedis)*

- Papillomatosis cutis lymphostatica

- Lymphocyst, lymphorrhea

- Reduced function and mobility

- Reduced productivity, financial problems

- Negative body image, anxiety and depression

- Angiosarcoma†

*Most common.

†Rare (after median interval of $10.6 \mathrm{yr}$ )

space occurs. Many experts now suggest that all chronic edema involves lymphatic dysfunction. ${ }^{5}$ High protein concentration in the interstitial fluid causes chronic inflammation and fibrosis formation accompanied by adipose tissue deposits.

Transient pitting unilateral or bilateral edema is characteristic of early stages of lymphedema. As the disease progresses, the pitting component gradually subsides and the skin becomes thick and rigid. Nonpitting edema, which was one of the findings in our patient, heralds the onset of fibrosis and irreversible skin changes (Box 2). ${ }^{6}$

Chronic lymphedema is a progressive, incurable and potentially disabling condition with serious complications (Box 3). 2,4,5,10 The most common potentially life-threatening complication is cellulitis or erysipelas. ${ }^{2,6,8}$

Prospective and retrospective studies show that lymphedema has a substantial negative long-term impact on physical, emotional and psychosocial well-being. ${ }^{2,9,10}$ Our patient's functional limitations from mobility and balance impairment are consistent with the findings of these studies. ${ }^{2}$

\section{Diagnosis}

Diagnosis of lower-limb lymphedema unrelated to cancer presents challenges and therefore it is often poorly recognized. ${ }^{2,10}$ The International Society of Lymphology consensus document, based on international protocols and practices, indicates that in most patients an accurate diagnosis of lymphedema can be predominantly based on the medical history and physical examination. ${ }^{3}$

Inspection, depending on the stage, typically shows pitting or nonpitting edema, cutaneous fibrosis, peau d'orange and a positive Stemmer sign (Box 1). The presence of cutaneous and subcutaneous thickening (pachydermia) strongly suggests the lymphatic pathogenesis of edema formation.

As a correct diagnosis is crucial for appropriate therapy, imaging studies should be considered if the diagnosis remains unclear, lymphoscintigraphy being the method of choice. ${ }^{3}$ Some cases of lymphedema require indirect radiocontrast lymphangiography to visualize lymph collecting vessels.

Our patient's history of long-standing edema in the legs and typical clinical presentation with pachydermia and fibrotic skin changes, as well as exclusion of other edema-causing pathologies and damage to the lymph vessels or nodes, is characteristic of primary lymphedema stage 3. Indirect lymphangiography was performed to visualize lymphatic vessels and confirm the diagnosis of lymphedema.

The differential diagnosis of lymphedema includes deep vein thrombosis, chronic venous insufficiency and lipedema.

\section{Treatment}

Although there is no cure for lymphedema, it can be managed successfully. The current standard of care for primary and secondary lymphedema includes complete decongestive therapy, which involves an initial intensive regimen of manual lymphatic drainage, exercise, skin care and use of compression bandages, followed by long-term maintenance therapy ${ }^{3,4,8}$ (Box 2).

A systematic review found moderately strong evidence supporting the effectiveness of complete decongestive therapy in treatment of lymphedema. ${ }^{11}$ A prospective study that included 299 patients with primary or secondary lymphedema reported volume reduction of $59.1 \%$ for participants with upper-extremity lymphedema and $62.7 \%$ for participants with lower-extremity lymphedema after an average 15.7 days of complete decongestive therapy..$^{12}$ At an average 9-month follow-up, the study showed that $84 \%$ of participants with upperextremity lymphedema and $82 \%$ of those with lower-extremity lymphedema were adherent to a self-management routine and maintained $90 \%$ of their initial volume reduction. Those who were nonadherent lost about one-third of the initial volume reduction. ${ }^{12}$

Early diagnosis and treatment of lymphedema, before irreversible skin changes and adipose tissue deposits develop, is key to effective volume reduction and prevention of complications. ${ }^{2,3}$ Early treatment can also shorten and simplify the course of treatment. We likely achieved a better and faster volume reduction in the right leg of our patient, as its treatment was started at an earlier stage of illness (Figure 5).

As a chronic condition, lymphedema is best treated using the chronic disease model that emphasizes the importance of selfmanagement with professional support. ${ }^{10}$ Satisfactory long-term control of our patient's bilateral leg volume with low-stretch compression stockings and exercises shows the efficacy of this model. We note, however, that our patient was highly adherent to the treatment during the intensive phase and to the self-management routine long term.

Full benefits of complete decongestive therapy can be achieved only when all 4 treatment components (compression, manual lymphatic drainage, skin care and exercise) are applied. Compression is often considered the most essential modality that effectively increases both venous and lymphatic drainage from the extremity. ${ }^{8}$ However, usually a better edema reduction is achieved with inclusion of manual lymphatic drainage in the treatment plan. An exploratory pilot study shows that this technique improves contractile lymphatic transport function. ${ }^{13}$ Manual lymphatic drainage also promotes edema reduction by directing lymph from the region of afflicted lymphatic vessels toward existing functional lymphatic structures. Good skin hygiene, exercise and prevention of infection are also important in the management of lymphedema.

Complete decongestive therapy, as well as posttreatment compression garments, should be prescribed by a physician or nurse practitioner and performed by a trained lymphedema therapist, 
Box 4: Contraindications for treatment of lymphedema ${ }^{1,9}$

Contraindications for manual lymphatic drainage

Absolute

- Decompensated heart failure

- Acute infections

- Untreated malignant diseases

- Deep vein thrombosis

Relative

- Asthma*

- Low blood pressure

- Thyroid disorder

Contraindications for compression therapy

- Severe arterial insufficiency

- Severe peripheral neuropathy

*Bronchoconstriction can result from the sympatholytic effect of manual lymphatic drainage.

using appropriate compression. ${ }^{3}$ The provider must be trained in complete decongestive therapy, as there are contraindications to both manual lymphatic drainage and compression (Box 4) (for more information, see https://canadalymph.ca/certified-lymphedema -therapists or https://canadalymph.ca/health-professionals/ schools). Treatment provided by untrained medical professionals entails considerable risk of serious complications and may lead to unnecessary expense and treatment failure. ${ }^{3}$ The coverage of decongestive therapy components in Canada varies from province to province (for more information, see https://canadalymph.ca/ wp-content/uploads/2015/04/CHART-Provincial-Coverage-Survey -results-March-2017.pdf).

Physicians often routinely prescribe diuretics to patients with lymphedema. Diuretics extract water from the edematous tissue, increasing protein concentration in the interstitium and promoting fibrosis formation. They also may induce water and electrolyte imbalance; therefore, their long-term administration is discouraged. ${ }^{3}$

\section{Conclusion}

Applied by a skilled therapist, complete decongestive therapy shows excellent results in lymphedema treatment. As the treatment outcome depends on the stage and severity of lymphedema, early diagnosis is important. The delay in diagnosis and appropriate treatment caused substantial changes in the size and structure of our patient's legs, accompanied by severe functional impairment, chronic discomfort and psychological distress.

\section{References}

1. Wittlinger $\mathrm{H}$, Wittlinger $\mathrm{D}$, Wittlinger $\mathrm{A}$, et al. Dr. Vodder's manual lymph drainage: a practical guide. New York: Thieme; 2011.

2. Shallwani SM, Hodson P, Towers A. Comparison between cancer-related and noncancer-related lymphedema: an overview of new patients referred to a specialized hospital-based center in Canada. Lymphat Res Biol 2017;15:64-9.

3. Executive Committee. The diagnosis and treatment of peripheral lymphedema: 2016 consensus document of the international society of lymphology. Lymphology 2016;49:170-84.

4. Weissleder $\mathrm{H}$, Schuchhardt C, editors. Lymphedema: diagnosis and therapy. 4th ed. Essen (Germany): Viavital Verlag; 2008.

5. Mortimer PS, Rockson SG. New developments in clinical aspects of lymphatic disease. J Clin Invest 2014;124:915-21.

6. Grada AA, Phillips TJ. Lymphedema: pathophysiology and clinical manifestations. J Am Acad Dermatol 2017;77:1009-20.

7. Rockson SG, Rivera KK. Estimating the population burden of lymphedema. Ann N Y Acad Sci 2008;1131:147-54.

8. Keast DH, Despatis M, Allen JO, et al. Chronic oedema/lymphoedema: underrecognised and under-treated. Int Wound J 2015;12:328-33.

9. Symvoulakis EK, Anyfantakis DI, Lionis C. Primary lower limb lymphedema: a focus on its functional, social and emotional impact. Int J Med Sci 2010;7:353-7.

10. Keast DH, Moffat C, Janmohammad A. Lymphedema impact and prevalence international study: the Canadian data. Lymphat Res Biol 2019;17:178-86.

11. Lasinski BB, McKillip Thrift K, Squire D, et al. A systematic review of the evidence for complete decongestive therapy in the treatment of lymphedema from 2004 to 2011. PM R 2012;4:580-601.

12. Ko DS, Lerner R, Klose G, et al. Effective treatment of lymphedema of the extremities. Arch Surg 1998;133:452-8.

13. Tan IC, Maus EA, Rasmussen JC, et al. Assessment of lymphatic contractile function after manual lymphatic drainage using near-infrared fluorescence imaging. Arch Phys Med Rehabil 2011;92:756-64.e1.

\section{Competing interests: None declared.}

This article has been peer reviewed.

The authors have obtained patient consent.

Affiliations: Alta Vista Chiropractic \& Massage Clinic (Bondalevich), Ottawa, Ont.; Vodder Schools International (Bondalevich), North Vancouver, BC; Department of Family Medicine (de Laplante), University of Ottawa, Ottawa, Ont.
Contributors: Larysa Bondalevich conceived the idea for the manuscript and Stefan de Laplante acquired clinical data for the work. Both authors designed and drafted the manuscript, revised it critically for important intellectual content, gave final approval of the version to be published and agreed to be accountable for all aspects of the work.

Correspondence to: Stefan de Laplante, Stefan.delaplante@ esfam.ca 